\title{
Proactive interference does not meaningfully distort visual working memory capacity estimates in the canonical change detection task
}

\author{
Po-Han Lin ${ }^{1}$ and Steven J. Luck ${ }^{2 *}$ \\ ${ }^{1}$ Graduate Institute of Learning and Instruction, National Central University, Jhongli, Taiwan \\ 2 Department of Psychology, Center for Mind and Brain, University of California Davis, Davis, CA, USA
}

Edited by:

Mattie Tops, University of Leiden, Netherlands

\section{Reviewed by:}

Ilja G. Sligte, University of

Amsterdam, Netherlands

Katherine Bettencourt, Harvard

University, USA

Mowei Shen, Zhejiang University,

China

*Correspondence:

Steven J. Luck, Center for Mind and Brain, University of California Davis, 267 Cousteau Place, Davis, CA 95618, USA.

e-mail: sjluck@ucdavis.edu
The change detection task has become a standard method for estimating the storage capacity of visual working memory. Most researchers assume that this task isolates the properties of an active short-term storage system that can be dissociated from long-term memory systems. However, long-term memory storage may influence performance on this task. In particular, memory traces from previous trials may create proactive interference that sometimes leads to errors, thereby reducing estimated capacity. Consequently, the capacity of visual working memory may be higher than is usually thought, and correlations between capacity and other measures of cognition may reflect individual differences in proactive interference rather than individual differences in the capacity of the short-term storage system. Indeed, previous research has shown that change detection performance can be influenced by proactive interference under some conditions. The purpose of the present study was to determine whether the canonical version of the change detection task - in which the to-be-remembered information consists of simple, briefly presented features - is influenced by proactive interference. Two experiments were conducted using methods that ordinarily produce substantial evidence of proactive interference, but no proactive interference was observed. Thus, the canonical version of the change detection task can be used to assess visual working memory capacity with no meaningful influence of proactive interference.

Keywords: working memory, proactive interference, capacity, change detection

\section{INTRODUCTION}

Visual working memory capacity has been studied extensively with variants of the change detection paradigm (Phillips, 1974; Luck and Vogel, 1997; Vogel et al., 2001; Woodman et al., 2001; Alvarez and Cavanagh, 2004; Olson and Jiang, 2004; Xu and Chun, 2006; Awh et al., 2007; Rouder et al., 2008). In this paradigm, a sample array containing several objects is presented briefly (e.g., $100 \mathrm{~ms}$ ), followed by a short retention interval (e.g., $900 \mathrm{~ms}$ ) and then a test array (see Figure 1). Observers report whether the test array is identical to the sample array or whether one item has changed. This task can readily be used to estimate an individual's working memory storage capacity. Specifically, if the number of items in the sample array (the set size, denoted $N$ ) exceeds the number of items that can be held in memory (the storage capacity, denoted $K)$, then only a subset of the items in the sample array will be stored in memory (i.e., $K \div N$ of the items). By measuring the actual probability that given observer is able to detect the changes, along with the probability of false alarms on no-change trials, it is possible to provide a mathematically principled estimate of $K$ for that observer (reviewed by Rouder et al., 2011). Strikingly, individual differences in $K$ measured from change detection and related tasks with very simple stimuli (e.g., colored squares) are strongly correlated with measures of complex cognitive abilities and global fluid intelligence (Fukuda et al., 2010; Gold et al., 2010).
Most research using this class of tasks has assumed, whether implicitly or explicitly, that performance reflects the active maintenance of information in a short-term memory system that can be distinguished from long-term memory systems (see review by Luck, 2008). Indeed, event-related potential (ERP) and functional magnetic resonance imaging (fMRI) studies have shown that neural activity increases temporarily during the retention interval in these tasks in a manner that is closely tied to individual differences in storage capacity (Vogel and Machizawa, 2004; Todd and Marois, 2005; Xu and Chun, 2006). However, the history of memory research is filled with examples of results that were initially attributed to a separate short-term memory system but were later explained by the same memory mechanisms that underlie long-term memory (see reviews by Crowder, 1982; Baddeley, 1986; Suprenant and Neath, 2009). In particular, limits in memory performance that were initially attributed to limits in storage capacity were later found to be explainable by the same interference mechanisms that explain failures of retrieval in long-term memory.

Two main types of interference might explain the apparent limits on visual working memory capacity in change detection paradigms. First, items that are similar to each other might be confused (see, e.g., the classic study of Conrad, 1964). In the case of long-term memory, for example, someone who is trying to 
A

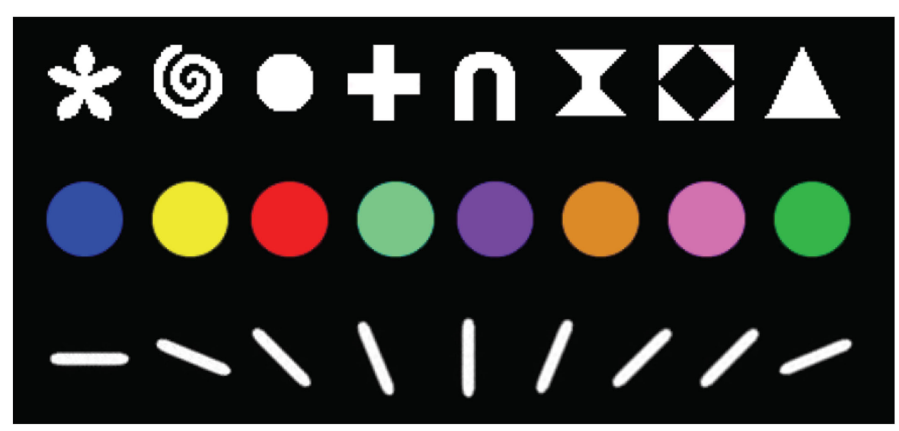

B

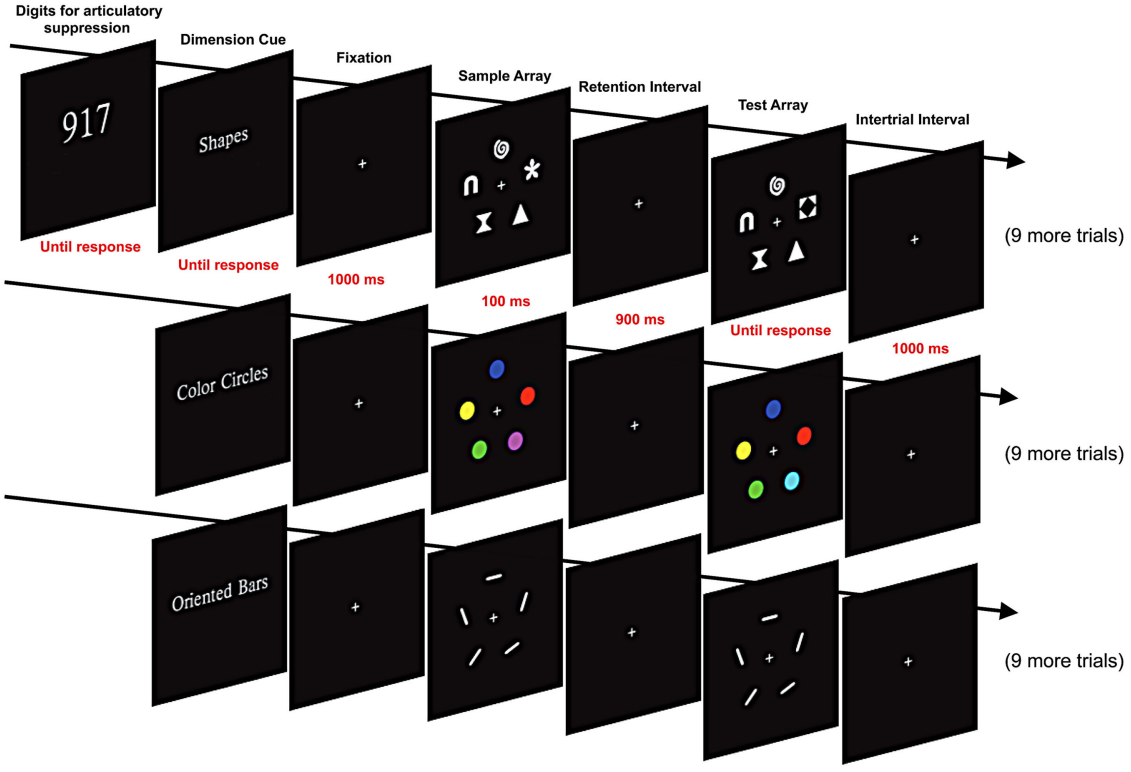

FIGURE 1 | Stimuli and design used in Experiment 1. (A) Shape, color, and orientation stimulus sets. (B) Sequence of events in a set of three blocks of trials. A set of three digits was presented at the beginning of each set of three blocks, and participants continuously repeated these digits aloud for the entire set of three blocks. Each block began with a cue indicating which feature set would be used for that block. This was followed with 10 change detection trials with the cued feature set. remember the name of a chemical that is highly similar to the names of other chemicals that were encoded at the same time might simply report the wrong chemical name at the time of test. Similarly, an individual who is trying to remember several similar colors in a change detection task might mix up the colors when the test array is presented. However, recent evidence suggests that this kind of interference plays little or no role in limiting performance in typical change detection tasks. First, Awh et al. (2007) found that change detection performance for Chinese characters was no worse for arrays containing four Chinese characters that it was for arrays containing two Chinese characters and two shaded cubes, despite the greater degree of interitem similarity when all four items were Chinese characters. (They did find that performance was worse when the similarity between sample and test stimuli was increased, but this is an inevitable consequence of decreased discriminability; that is, a small enough change will always be difficult to detect.) Second, Lin and Luck (2008) found that color change detection performance was no worse for arrays containing three highly similar colors than it was for arrays containing three very different colors. Thus, there is no evidence that interitem similarity has a significant impact on change detection performance, which contrasts with the substantial effects of interitem similarity that have been well-documented in the long-term memory literature (Tulving and Psotka, 1971; Runquist, 1975; Anderson and Neely, 1996).

A second type of interference might limit performance in visual change detection tasks, namely proactive interference from items presented on previous trials. Proactive interference usually arises when participants are confused about whether an item was presented on the current trial or on a previous trial. For example, in the classic study of Peterson and Peterson (1959), participants received a list of three letters, which they were then required to recall after a filled delay period. Recall performance became gradually less accurate as the delay period increased, nearing floor by a delay of $18 \mathrm{~s}$, and this was originally attributed to a gradual fading of a short-term memory representation. However, it could also be explained by a confusion between the letters that had been presented at the beginning of the current trial and the letters that had 
been presented on previous trials. For example, Keppel and Underwood (1962) showed that recall was very good at long delays on the first trial (presumably because there was no interference from previous trials). Similarly, Wickens et al. (1963) presented participants with several trials with one class of memory materials and then switched to another class, and they found no evidence of decay for the first trial with a new type of memory material (presumably because the different type of material from the previous trials did not cause interference with the current trial). Similar effects have been observed in complex working memory span tasks (Bunting, 2006). This recovery of performance for the first trial of a new type of material is called release from proactive interference.

This same type of interference could, in principle, arise in change detection tasks. For example, if an item was blue in the sample array and changed to red in the test array, the presence of red on a previous trial might lead to a feeling of familiarity for the red item in the current trial, and the observer might therefore not report that the red color is new (for the current trial). Indeed, proactive interference has been shown to have a significant impact on estimates of working memory capacity in complex span tasks (May et al., 1999), and individual differences in the ability to overcome proactive interference may explain some of the correlations between complex span performance and measures of cognitive ability (Lustig et al., 2001; Unsworth and Engle, 2007; Blalock and McCabe, 2011). Thus, it is critically important to determine whether measures of capacity from visual change detections tasks - which are also strongly correlated with measures of cognitive ability - might be distorted by proactive interference.

Two studies suggest that this is a real possibility. First, Makovski and Jiang (2008) found that observers were less likely to detect a change if the changed color on the current trial had been present on the previous trial. However, this effect was present only if this color had been at the same location on the previous trial. Although this is theoretically interesting, such trials are relatively uncommon, so they probably do not have much impact on working memory capacity estimates.

Hartshorne (2008) also found evidence of proactive interference. In one experiment, complex objects were presented in the sample array, and the test array was a single object in the center; participants were instructed to indicate whether this test item had been in the sample array. They were much more likely to make a false alarm (reporting a match when the test item had not been in the sample array) if the test item had been present in one of the three previous trials. In a second experiment, different stimulus sets were used (e.g., sets of colored squares, sets of geometric objects), and 10 consecutive trials were tested with a given set, followed by 10 trials with a new set. Participants were approximately $4 \%$ more accurate on the first trial in a set of 10 in the in the last trial, suggesting that interference had built up over the 10 trials. In a third experiment, over 3000 participants were tested in a shape change detection task via an Internet site, making it possible to examine performance on each of the first 10 individual trials. Estimated memory capacity $(K)$ dropped by approximately $1 / 4$ of an item between the first and second trials, suggesting that performance was impacted by interference after the first trial. These results indicated that the strict limits on visual working memory capacity cannot be completely explained by proactive interference, but interference can impact estimated working memory capacity by as much as $15 \%$.

Although these interference effects are small on average, they may be considerably larger in some participants than in other, so individual differences in performance might reflect individual differences in the ability to inhibit interference from previous trials. Consequently, it is conceivable that correlations between working memory capacity (as estimated from change detection performance) and other cognitive measures could reflect individual differences in the inhibition of interference from long-term memory representations. This is of great theoretical importance, because it would mean that the correlations do not reflect individual differences in the use of an active short-term memory storage system.

Although the results presented by Hartshorne (2008) clearly demonstrate that some variants of the change detection task can be impacted by proactive interference, there are reasons to believe that the "canonical" version of this paradigm introduced by Luck and Vogel (1997) may be less prone to proactive interference. In the Luck and Vogel (1997) version, the sample array consisted of simple features (colored squares or colored, oriented bars) that were presented for only $100 \mathrm{~ms}$, followed by a $900-\mathrm{ms}$ retention interval and then a 2000-ms test display. A 2500-ms intertrial interval separated trials (for details, see Vogel et al., 2001). The brief duration of the sample display was designed to simulate the brief period of time that visual information is available during a typical fixation period in natural scene viewing (Henderson, 2008), and the combination of the brief sample duration and brief retention interval were designed to minimize opportunities for storing the information in long-term memory. In contrast, most of the stimuli used by Hartshorne (2008) were relatively complex shapes, and the sample array duration was $1000 \mathrm{~ms}$. This may have provided more opportunity for long-term memory representations to be created. The same timing was used by Makovski and Jiang (2008), who also found evidence of interference from the previous trial. Consequently, it is possible that proactive interference impacted performance more in these studies than would be found in the Luck and Vogel (1997) version of the task. This does not imply that the proactive interference effects in the Hartshorne (2008) and Makovski and Jiang (2008) studies are artifactual or uninteresting. However, it suggests that relatively pure estimates of visual working memory capacity can be obtained under conditions that minimize proactive interference.

The purpose of the present study was therefore to determine whether the canonical Luck and Vogel (1997) version of the change detection task provides a pure measure of the storage capacity of a short-term memory system, with negligible contamination from proactive interference. A few changes were made to the canonical version (e.g., using articulatory suppression rather than a verbal memory load to minimize contributions from verbal working memory). However, three key elements were retained: (a) the use of simple stimuli; (b) a very brief sample array duration; and (c) a relatively brief retention interval. These are also the conditions under which ERP studies have shown that an active representation is still available at the time of the test array (Vogel and Machizawa, 2004; Vogel et al., 2005). 


\section{EXPERIMENT 1}

Experiment 1 took the approach pioneered by Wickens et al. (1963) and also used by Hartshorne (2008), in which different classes of stimuli are used and the class is changed every 10 trials. As shown in Figure 1, the stimuli on a given trial were colored squares, oriented lines, or shapes. Participants experienced short blocks of 10 trials with one of these three classes (preceded by a cue at the beginning of each block to indicate what type of stimuli would be presented for the next 10 trials). If proactive interference impacts change detection performance, then performance should be best on the first trial of a block and then drop over the next few trials. Performance should then recover on the first trial of the next block owing to release from proactive interference. This is the pattern that was observed by Wickens et al. (1963) for verbal material and by Hartshorne (2008) in a visual change detection task. However, if proactive interference is minimized by the use of simple stimuli, brief sample array presentations and a short retention interval, then performance should be constant over the course of each block.

\section{METHODS}

\section{Participants}

Thirty undergraduate students between the ages of 18 and 30 participated for course credit. They reported no history of neurological problems and reported having normal color vision and normal or corrected-to-normal visual acuity.

\section{Stimuli}

Stimulus array were presented within a $4.91^{\circ} \times 4.91^{\circ}$ region on a video monitor with a black background, viewed at a distance of $70 \mathrm{~cm}$. Each array contained five items. Each item was placed in one of five pre-specified locations on an imagery circle $\left(\right.$ radius $=1.95^{\circ}$ ), and items were separated from each other by $2.29^{\circ}$ (center-to-center). A fixation cross was visible throughout each trial block. The chromaticity and luminance values for the stimuli were measured with a Tektronix model J17 colorimeter using the 1976 CIE color coordinate system. Eight stimulus colors were used: red $\left(u^{\prime}=0.467, v^{\prime}=0.520 ; 21.85 \mathrm{~cd} / \mathrm{m}^{2}\right)$, pink $\left(u^{\prime}=0.301, v^{\prime}=0.421 ; 36.09 \mathrm{~cd} / \mathrm{m}^{2}\right)$, blue $\left(u^{\prime}=0.177, v^{\prime}=0.167\right.$; $\left.10.76 \mathrm{~cd} / \mathrm{m}^{2}\right)$, green $\left(u^{\prime}=0.298, v^{\prime}=0.576 ; 65.71 \mathrm{~cd} / \mathrm{m}^{2}\right)$, yellow $\left(u^{\prime}=0.230, v^{\prime}=0.546 ; 74.62 \mathrm{~cd} / \mathrm{m}^{2}\right)$, violet $\left(u^{\prime}=0.233\right.$, $\left.v^{\prime}=0.235 ; \quad 18.74 \mathrm{~cd} / \mathrm{m}^{2}\right), \quad$ orange $\quad\left(u^{\prime}=0.356, \quad v^{\prime}=0.533\right.$; $\left.32.88 \mathrm{~cd} / \mathrm{m}^{2}\right)$, and teal $\left(u^{\prime}=0.159, v^{\prime}=0.315 ; 32.63 \mathrm{~cd} / \mathrm{m}^{2}\right)$.

As illustrated in Figure 1A, three sets of distinct feature dimensions were used: color, shape, and orientation. On color trials, five colored circles were presented, each subtending $1.00^{\circ} \times 1.00^{\circ}$ of visual angle, with a color that was randomly selected (without replacement) from the set of eight colors described above. On shape trials, five items were randomly selected (without replacement) from a set of eight shapes: triangle, gateway, hourglass, circle, cross, flower, spiral, and black diamond within a white square. Each of these shapes was $1.04^{\circ} \times 1.04^{\circ}$ and drawn in white $\left(98.24 \mathrm{~cd} / \mathrm{m}^{2}\right)$. On orientation trials, oriented bars $\left(1.53^{\circ} \times 0.26^{\circ}\right.$, $98.24 \mathrm{~cd} / \mathrm{m}^{2}$ ) were randomly selected (without replacement) from a set of eight orientations: $0^{\circ}, 22.5^{\circ}, 45.0^{\circ}, 67.5^{\circ}, 90.0^{\circ}, 112.5^{\circ}$, $135.0^{\circ}$, or $157.5^{\circ}$. Note that no color, shape, or orientation could appear more than once in an array, and when an object changed, it changed to a value that was not present in the sample array.

\section{Procedure}

Each trial consisted of a 100-ms sample array followed by a $900-$ ms blank fixation delay period and then a test array that stayed on until the participant responded (see Figure 1B). The sample and test arrays were identical except that one feature (e.g., color, shape, or orientation) of one item in the test array was different from that of the corresponding item in the sample array on $50 \%$ of the trials. Participants responded by pressing one of two buttons on each trial to indicate whether the sample and test arrays were the same or different. Accuracy was stressed rather than speed, and the responses were not timed.

Each participant was tested in a single session of 30-45 min, including rest breaks. The experiment was divided into 10 sets of 3 trial blocks, with 10 trials using a single feature type in each block. Each set of three trial blocks included one block with each of the three feature sets (e.g., 10 trials with shape, then 10 trials with color, and then 10 trials with orientation). The order of feature sets was constant for a given participant but counterbalanced across participants. The change and no-change trials were randomly distributed across the total set of 300 trials.

Participants were informed of the feature set that would be used in a given block by means of a cue at the beginning of the block (see Figure 1B). The cue was visible until the participant initiated the block by pressing a button.

To minimize contributions from verbal working memory, we used an articulatory suppression procedure in which three randomly selected white digits $\left(2.67^{\circ} \times 3.41^{\circ}\right)$ were presented side by side at fixation at the beginning of each set of three blocks (see Figure 1B). Participants were instructed to repeat these three digits aloud throughout each block of trials (e.g., "nine-one-sevennine-one-seven..."). Previous research has shown that this is an effective method for discouraging verbal encoding of visual stimuli (Besner et al., 1981; Baddeley, 1986).

\section{RESULTS AND DISCUSSION}

In this experiment, proactive interference should lead to a progressive decline in performance across trials within a block of 10 trials with the same stimulus set, and release from proactive interference should lead to a rebound in performance on the first trial of a block. To assess these patterns, we grouped all of the trials for a given sequential position within a block, combining across the 30 blocks (and therefore averaging across the three feature types). This yielded 30 trials per position for each participant.

Figure 2 shows mean change detection accuracy for each of the 10 sequential positions. Accuracy was approximately equal across all 10 sequential positions, with mean accuracy ranging between 69 and 75\% correct. A sharp decline in performance over the first few trials was not observed, nor was an improvement observed on the first trial of a block compared to the last several trials. A one-way within-subjects analysis of variance (ANOVA) indicated that there were no significant differences among the 10 sequential positions, $[F(9,261)=1.80, p=0.0687]$. As shown in Figure 2, the pattern of means did not follow the typical proactive interference pattern, in which accuracy should be high in the first 


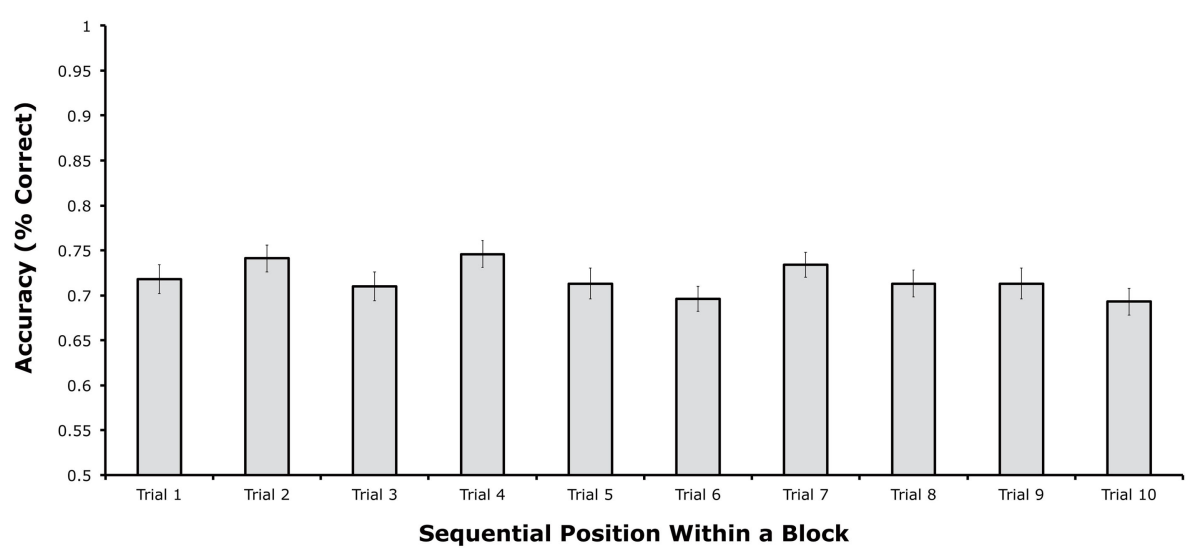

FIGURE 2 | Mean change detection accuracy from each of the 10 sequential positions within a block in Experiment 1 (error bars show $95 \%$ within-subjects confidence intervals, as described by Loftus and Loftus, 1988).

sequential position and then drop to an asymptote over the next few positions. Instead, accuracy bounced up (at positions 2, 4, and 7) and down (e.g., at positions 3, 5, and 6) in a non-systematic manner.

To test for the specific pattern expected from proactive interference, we conducted a follow-up $t$-test comparing the first position with the average of the last three positions. This analysis did not approach significance $[t(58),=0.476, p=0.6359]$. We also performed an ANOVA on the first three sequential positions, but again no significant differences were found, $F(2,58)=1.429, p=0.2479$. Thus, if there were any differences among positions, they did not follow the typical pattern of proactive interference. For example, in an experiment that also involved a switch in stimulus class every 10 trials, Hartshorne (2008, Experiment 2) found significantly better performance on the first of trial of a given stimulus class than on the last trial, which is the expected proactive interference pattern. In contrast, we found nearly equivalent performance between the beginning and end of each block of 10 trials. Thus, it is likely that the non-significant and non-systematic differences among sequential positions reflect random variation.

It is impossible to prove the null hypothesis with conventional statistical tests. We therefore performed two additional analyses that can indicate the likelihood that there were no meaningful proactive interference effects in these data (despite a relatively large sample size). First, we used the Bayes Factor approach, which can convert a $t$ value into the relative likelihood of the null and alternative hypotheses (Rouder et al., 2009 - online calculator available at http://pcl.missouri.edu/bayesfactor). When applied to the difference in accuracy between the first sequential position and the average of the last three sequential positions, this analysis indicated that the null hypothesis was 6.34 times more likely to be true than the alternative hypothesis. Second, we computed the difference in accuracy between the first sequential position and the average of the last three sequential positions for each participant and conducted a confidence interval analysis. This analysis indicated that the mean difference was $1.1 \%$, with a $95 \%$ confidence interval of $\pm 3.1 \%$. Thus, we can be $95 \%$ confident that accuracy for the first sequential position was no more than $4.2 \%$ greater than the average accuracy across the last three sequential positions. Together, these analyses indicate that the null hypothesis was much more likely to be true than the hypothesis of a proactive interference effect, and even if there was a proactive interference effect, it was very small (much smaller than the effect observed by Hartshorne, 2008).

\section{EXPERIMENT 2}

The goal of Experiment 2 was to generalize the results of Experiment 1 and rule out a potential explanation for the lack of any evidence of proactive interference. Specifically, a task switching cost in Experiment 1 may have impaired performance for the first few trials of each block, offsetting a release from proactive interference that would otherwise have been visible. Many previous studies of proactive interference using this approach have observed release from proactive interference (greater accuracy on the first few trials of each block) despite having this same type of task switching (e.g., Wickens et al., 1963; Hartshorne, 2008). However, it is nonetheless important to rule out the possibility that a small proactive interference effect was present in Experiment 1 but was masked by a small task switching cost.

To assess this possibility, we conducted a session entirely using color change detection, but we made one set of colors very common and another set very rare (see Figure 3 ). Trials with the rare color set were separated by an average of 19 trials using the frequent color set, which should have virtually eliminated any proactive interference for the rare color set. Conversely, the vast majority of trials with the frequent color set were preceded by several trials with the same set of colors, which should have maximized proactive interference. If proactive interference contributes significantly to performance in this paradigm, then participants should be much less accurate for the frequently occurring color set than for the infrequently occurring color set.

\section{METHOD}

\section{Participants}

A new group of 20 undergraduate students from the same pool participated for course credit. These participants reported no history of neurological problems and reported having normal color vision and normal or corrected-to-normal visual acuity. 
A

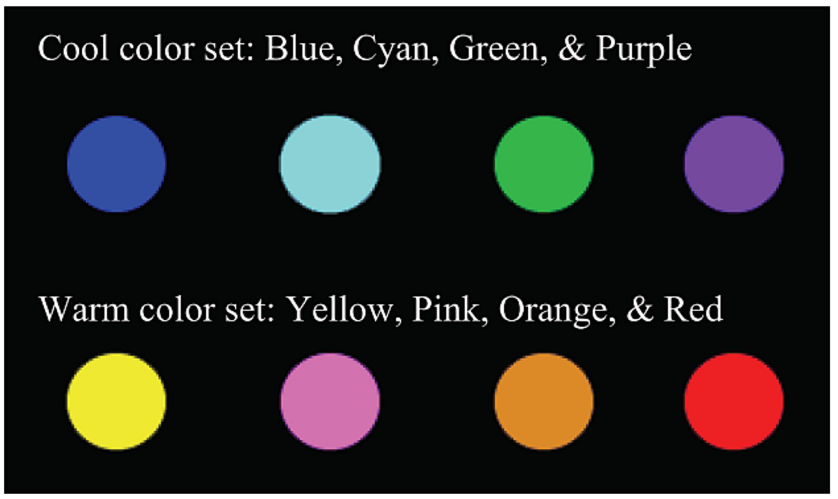

B

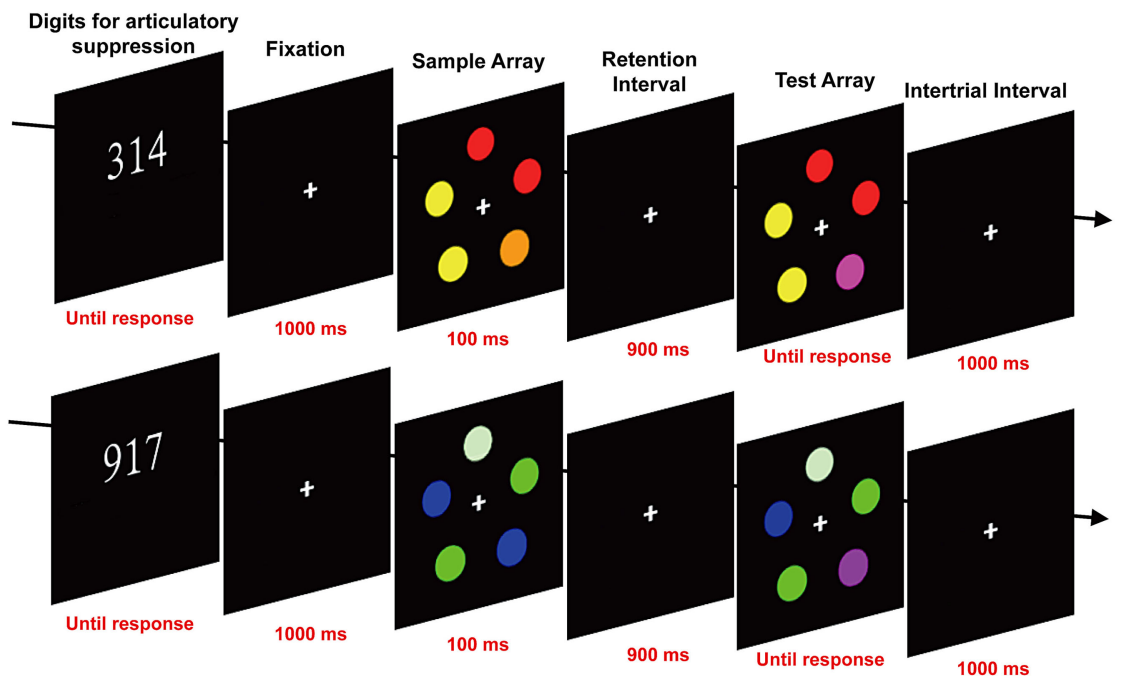

C

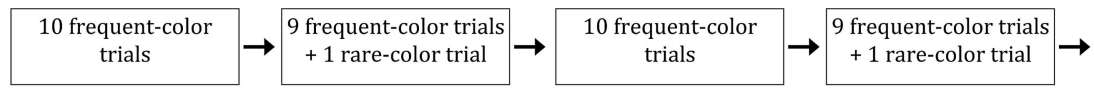

FIGURE 3 | Stimuli and design used in Experiment 2. (A) Cool and warm color sets. (B) Sequence of events on a typical single trial with each color set. (C) Method for ensuring that each trial using the rare color set was separated by $10-28$ trials using the frequent color set.

\section{Stimuli}

The stimuli were the same as those in the color condition of Experiment 1, except that different colors were used. Colors were separated into two sets, each with four values. The warm color set consisted of red $\left(u^{\prime}=0.467, v^{\prime}=0.520 ; 21.85 \mathrm{~cd} / \mathrm{m}^{2}\right)$, pink $\left(u^{\prime}=0.301, v^{\prime}=0.421 ; 36.09 \mathrm{~cd} / \mathrm{m}^{2}\right)$, orange $\left(u^{\prime}=0.356\right.$, $\left.v^{\prime}=0.533 ; 32.88 \mathrm{~cd} / \mathrm{m}^{2}\right)$, and yellow $\left(u^{\prime}=0.230, v^{\prime}=0.546\right.$; $\left.74.62 \mathrm{~cd} / \mathrm{m}^{2}\right)$. The cool color set consisted of blue $\left(u^{\prime}=0.176\right.$, $\left.v^{\prime}=0.167 ; \quad 11.32 \mathrm{~cd} / \mathrm{m}^{2}\right), \quad$ green $\quad\left(u^{\prime}=0.128, \quad v^{\prime}=0.556\right.$; $\left.69.19 \mathrm{~cd} / \mathrm{m}^{2}\right)$, violet $\left(u^{\prime}=0.233, v^{\prime}=0.235 ; 18.74 \mathrm{~cd} / \mathrm{m}^{2}\right)$, and cyan $\left(u^{\prime}=0.173, v^{\prime}=0.444 ; 50.28 \mathrm{~cd} / \mathrm{m}^{2}\right)$. As illustrated in Figure $3 \mathrm{~A}$, the two color sets were very distinct from each other, and yet the colors within each set could easily be discriminated.

On any given trial, the colors of the five items were selected at random from either the cool set or the warm set, but with the constraint that no color could appear more than two times in a single array. This constraint was necessary to maintain a set size of five items (thereby avoiding ceiling and floor effects) without having so many different colors within a set that they would be difficult to discriminate from each other. The small number of colors in each set also increased the likelihood that the colors used on one trial of the frequent set had been repeated multiple times over the past few trials. When a change occurred, the change was always to a different member of the same set.

\section{Procedure}

The procedure was the same as in Experiment 1 (see Figure 3B), except as follows. For each subject, one of the two color sets was frequent and the other was rare (this assignment was counterbalanced across subjects). The frequent set occurred on $95 \%$ of trials and the rare set occurred on $5 \%$ of trials. Between 10 and 28 trials with the frequent color set were interposed between each trial with the rare color set. This was achieved by dividing the sequence of trials into alternating groups of 10 trials: one group in which all 10 trials used the frequent color set followed by one group in which 9 of the 10 trials used the frequent set 
and 1 randomly selected trial used the rare set (see Figure 3C). Each participant was tested in a single session of 32 blocks of 20 trials, with a break between each block. This yielded a total of 32 trials of the rare color set and 608 trials of the frequent color set.

\section{RESULTS AND DISCUSSION}

Accuracy was computed separately for trials using the rare and frequent color sets, disregarding which colors were rare or frequent for a given participant. As shown in Figure 4, mean accuracy was very similar for trials using the rare and frequent color sets. A paired $t$-test indicated that there was no significant difference between these trial types, $t(18)=1.70, p=0.1063$, and the small and non-significant difference was actually in the direction of greater accuracy for the frequent color set. The Bayes factor analysis indicated that the null hypothesis was 1.60 times more likely to be true than the alternative hypothesis. This value was not as high as in Experiment 1, but this may simply reflect the small and non-significant difference in the direction opposite to the effect predicted by proactive interference.

We also performed a confidence interval analysis on the difference in accuracy between the two trial types (rare minus frequent). The mean difference score was $-3.5 \%$ with a $95 \%$ confidence interval of $\pm 3.8 \%$. In other words, we have $95 \%$ confidence that the effect of trial type was between -7.3 and $+0.3 \%$. Thus, we can be $95 \%$ certain that any impairment in performance due to repetition of a color set was less than $0.3 \%$.

These results are consistent with the hypothesis that memory performance in the canonical version of the change detection task depends virtually exclusively on memory for the current trial, with little or no impact of memory from preceding trials. Even when the same four colors were repeated trial after trial, these data indicate that memory was no more than $0.3 \%$ worse than memory for colors that appeared only infrequently. Thus, we can be confident that proactive interference does not meaningfully distort estimates of working memory capacity in this variant of the change detection paradigm.

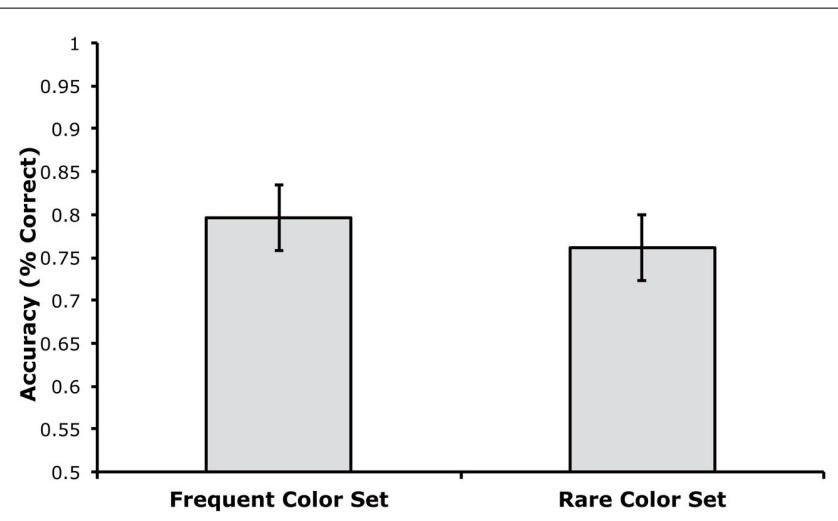

FIGURE 4 | Mean change detection accuracy for trials with the frequent and rare color sets in Experiment 2 (error bars show 95\% within-subjects confidence intervals, as described by Loftus and Loftus, 1988).

\section{GENERAL DISCUSSION}

Previous research clearly indicates that long-term memory processes, including proactive interference, can sometimes lead to the appearance of capacity limitations (Crowder, 1982; Baddeley, 1986; Suprenant and Neath, 2009). Research by Makovski and Jiang (2008) and by Hartshorne (2008) has further shown that proactive interference can influence estimates of visual working memory capacity in the change detection paradigm. However, as noted by Hartshorne (2008), the effects of proactive interference on change detection in these studies were modest and cannot explain the very strict limits on visual working memory capacity observed in change detection experiments. The present study goes one step further, showing that proactive interference can be virtually eliminated when the stimuli are simple, the sample array is brief, and the retention interval is short. Although the present results do not indicate which of these factors are essential, they do indicate that it is possible to use these parameters to isolate working memory capacity, independent of proactive interference from long-term memory. Similar parameters were used in a study showing a very strong correlation $\left(r^{2}=0.79\right)$ between visual working memory capacity and performance on a broad battery of higher cognitive function (Gold et al., 2010), so it seems likely that this correlation was not driven by individual differences in proactive interference. Future studies linking visual working memory capacity with other cognitive processes would be well advised to use similar parameters as well.

More broadly, these results are consistent with the hypothesis that a short-term visual storage system exists that can be distinguished from long-term memory. The idea of separate short-term and long-term memory stores has been widely criticized in the context of verbal memory (Crowder, 1982; Ranganath and Blumenfeld, 2005; Suprenant and Neath, 2009). However, there is excellent evidence of active maintenance of visual information during the delay interval in change detection tasks (Vogel and Machizawa, 2004; Todd and Marois, 2005; $\mathrm{Xu}$ and Chun, 2006). A recent study also provided significant evidence of active maintenance of semantic information in working memory (Shivde and Anderson, 2011). The present results, together with those of Hartshorne (2008), indicate that the severe limits on visual working memory capacity cannot be explained by proactive interference. Similarly, Lin and Luck (2008) showed that similarity-based interference - which plays a major role in failures of long-term memory retrieval - has no significant effect on visual working memory performance in the canonical version of the change detection task. Together, these results provide converging evidence against a monolithic view of memory.

It is important to consider whether the lack of an effect of sequential position in Experiment 1 and the lack of a difference between the rare and frequent stimulus sets in Experiment 2 might reflect the presence of equivalent amounts of proactive interference across conditions rather than an absence of proactive interference. Although it is impossible to rule out this possibility, it seems very unlikely. First, the manipulation used in Experiment 1 has been found to produce substantial proactive interference effects in many previous studies (e.g., Wickens et al., 1963; Bunting, 2006; Hartshorne, 2008). There is no obvious reason why there 
would be no evidence of release from proactive interference in the present experiment when the same manipulation produced reliable effects in prior studies (except that the present version of the task is not influenced by proactive interference). Second, Experiment 2 used an extreme manipulation, in which trials using the frequent stimulus set were preceded by an average of 18 successive trials that used exactly the same set of four colors, which should have produced massive proactive interference (if proactive interference could influence performance in this task). In contrast, trials using the rare stimulus set were preceded by an average of 18 successive trials using a different stimulus set, which should have produced minimal proactive interference. If proactive interference could influence performance in the present task, it would be very surprising not to observe a difference in performance between these two extreme conditions. Thus, although it is never possible to prove the null hypothesis, the present experiments provide strong support for the proposal that proactive interference has very little effect on performance of the canonical version of the change detection task.

An important caveat is that long-term memory can certainly influence change detection performance under some conditions. With longer stimulus durations, Makovski and Jiang (2008) and Hartshorne (2008) found evidence of modest proactive

\section{REFERENCES}

Alvarez, G. A., and Cavanagh, P. (2004). The capacity of visual short-term memory is set both by information load and by number of objects. Psychol. Sci. 15, 106-111.

Anderson, M. C., and Neely, J. H. (1996). "Interference and inhibition in memory retrieval," in Memory, Handbook of Perception and Cognition, 2nd Edn, eds. E. L. Bjork and R. A. Bjork (San Diego: Academic Press), 237-313.

Awh, E., Barton, B., and Vogel, E. K. (2007). Visual working memory represents a fixed number of items regardless of complexity. Psychol. Sci. 18, 622-628.

Baddeley, A. D. (1986). Working Memory. Oxford: Clarendon.

Besner, D., Davies, J., and Daniels, S. (1981). Reading for meaning: the effects of concurrent articulation. $Q$. J. Exp. Psychol. 33A, 415-437.

Blalock, L. D., and McCabe, D. P. (2011). Proactive interference and practice effects in visuospatial working memory span task performance. Memory 19, 83-91.

Bunting, M. (2006). Proactive interference and item similarity in working memory. J. Exp. Psychol. Learn. Mem. Cogn. 32, 183-196.

Clapp, W. C., Rubens, M. T., and Gazzaley, A. (2010). Mechanisms of working memory disruption by external interference. Cereb. Cortex 20, 859-872.

Conrad, R. (1964). Information, acoustic confusion and mem- ory span. Br. J. Psychol. 55, 1964.

Crowder, R. G. (1982). The demise of short-term memory. Acta Psychol. (Amst.) 50, 291-323.

Fukuda, K., Vogel, E., Mayr, U., and Awh, E. (2010). Quantity, not quality: the relationship between fluid intelligence and working memory capacity. Psychon. Bull. Rev. 17, 673-679.

Gold, J. M., Hahn, B., Zhang, W., Robinson, B. M., Kappenman, E. S., Beck, V. M., and Luck, S. J. (2010). Reduced capacity but spared precision and maintenance of working memory representations in schizophrenia. Arch. Gen. Psychiatry 67, 570-577.

Hartshorne, J. K. (2008). Visual working memory capacity and proactive doi:10.1371/journal.pone.0002716

Henderson, J. M. (2008). "Eye movements and visual memory," in Visual Memory, eds S. J. Luck and A. Hollingworth (New York: Oxford University Press), 87-121.

Keppel, G., and Underwood, B. J. (1962). Proactive inhibition in short-term retention of single items. J. Verbal Learn. Verbal Behav. 1, 153-161.

Lin, P.-H., and Luck, S. J. (2008). The influence of similarity on visual working memory representations. Vis. Cogn. 17, 356-372.

Loftus, G. R., and Loftus, E. F. (1988). Essence of Statistics. New York: Random House. interference. PLOS ONE 3, e2716.

interference, suggesting that long-term memory can play a role when sufficient time is provided for long-term memory encoding. Similarly, patients with medial temporal lobe amnesia exhibit deficits on change detection with long retention intervals $(8 \mathrm{~s})$ but not short retention intervals ( $1 \mathrm{~s}$; Olson et al., 2006), suggesting that long-term memory mechanisms begin to play a role when the retention interval increases. In addition, in studies using relatively long encoding periods and retention intervals, Gazzaley and his colleagues have shown that information may initially be stored in an active form in working memory, then temporarily displaced while another task is being performed, and then be restored from long-term memory (Clapp et al., 2010). Thus, although change detection paradigms can isolate an active short-term storage system under some conditions, these paradigms can also be strongly influenced by long-term memory systems under other conditions. Considerable caution is therefore necessary when using change detection paradigms to isolate active, short-term memory storage mechanisms.

\section{ACKNOWLEDGMENTS}

This study was made possible by grant R01MH076226 to Steven J. Luck from the National Institute of Mental Health.

Luck, S. J. (2008). "Visual short-term memory," in Visual Memory, eds S. J. Luck and A. Hollingworth (New York: Oxford University Press), 43-85.

Luck, S. J., and Vogel, E. K. (1997). The capacity of visual working memory for features and conjunctions. Nature 390, 279-281.

Lustig, C., May, C. P., and Hasher, L. (2001). Working memory span and the role of proactive interference. $J$. Exp. Psychol. Gen. 130, 199-207.

Makovski, T., and Jiang, Y. V. (2008). Proactive interference from items previously stored in visual working memory. Mem. Cognit. 36, 43-52.

May, C. P., Hasher, L., and Kane, M. J. (1999). The role of interference in memory span. Mem. Cognit. 27, 759-767.

Olson, I. R., and Jiang, Y. (2004). Visual short-term memory is not improved by training. Mem. Cognit. $32,1326-1332$

Olson, I. R., Page, K., Moore, K. S., Chatterjee, A., and Verfaellie, M. (2006). Working memory for conjunctions relies on the medial temporal lobe. $J$. Neurosci. 26, 4596-4601.

Peterson, L. R., and Peterson, M. J. (1959). Short-term retention of individual verbal items. J. Exp. Psychol. 58, 193-198.

Phillips, W. A. (1974). On the distinction between sensory storage and short-term visual memory. Percept. Psychophys. 16, 283-290.

Ranganath, C., and Blumenfeld, R. S. (2005). Doubts about double dissociations between short- and long-term memory. Trends Cogn. Sci. (Regul. Ed.) 9, 374-380.

Rouder, J. N., Morey, R. D., Cowan, N., Zwilling, C. E., Morey, C. C., and Pratte, M. S. (2008). An assessment of fixed-capacity models of visual working memory. Proc. Natl. Acad. Sci. U.S.A. 105, 5975-5979.

Rouder, J. N., Morey, R. D., Morey, C. C., and Cowan, N. (2011). How to measure working memory capacity in the change detection paradigm. Psychon. Bull. Rev. 18, 324-330.

Rouder, J. N., Speckman, P. L., Sun, D., Morey, R. D., and Iverson, G. (2009). Bayesian t tests for accepting and rejecting the null hypothesis. Psychon. Bull. Rev. 16, 225-237.

Runquist, W. N. (1975). Interference among memory traces. Mem. Cognit. 3, 143-159.

Shivde, G., and Anderson, M. C. (2011). On the existence of semantic working memory: evidence for direct semantic maintenance. J. Exp. Psychol. Learn. Mem. Cogn. 37, 1342-1370.

Suprenant, A. M., and Neath, I. (2009). "The 9 lives of short-term memory," in Interactions Between Short-Term and Long-Term Memory in the Verbal Domain, eds A. Thorn and M. Page (Hove: Psychology Press), 16-43.

Todd, J. J., and Marois, R. (2005). Posterior parietal cortex activity predicts individual differences in visual short-term memory capacity. Cogn. Affect. Behav. Neurosci. 5, 144-155. 
Tulving, E., and Psotka, J. (1971). Retroactive inhibition in free recall: Inaccessibility of information available in the memory store. J. Exp. Psychol. 87, 1-8.

Unsworth, N., and Engle, R. W. (2007). The nature of individual differences in working memory capacity: active maintenance in primary memory and controlled search from secondary memory. Psychol. Rev. 114, 104-132.

Vogel, E. K., and Machizawa, M. G. (2004). Neural activity predicts individual differences in visual working memory capacity. Nature 428, 748-751.
Vogel, E. K., McCollough, A. W., and Machizawa, M. G. (2005). Neural measures reveal individual differences in controlling access to working memory. Nature 438, 500-503.

Vogel, E. K., Woodman, G. F., and Luck, S. J. (2001). Storage of features, conjunctions, and objects in visual working memory. J. Exp. Psychol. Hum. Percept. Perform. 27, 92-114.

Wickens, D. D., Born, D. G., and Allen, C. K. (1963). Proactive inhibition and item similarity in short-term memory. J. Verbal Learn. Verbal Behav. 2, 440-445.

Woodman, G. F., Vogel, E. K., and Luck, S. J. (2001). Visual search remains efficient when visual working memory is full. Psychol. Sci. 12, 219-224.

Xu, Y., and Chun, M. M. (2006). Dissociable neural mechanisms supporting visual short-term memory for objects. Nature 440, 91-95.

Conflict of Interest Statement: The authors declare that the research was conducted in the absence of any commercial or financial relationships that could be construed as a potential conflict of interest.

Received: 14 December 2011; accepted: 07 February 2012; published online: 28 February 2012.
Citation: Lin P-H and Luck SJ (2012) Proactive interference does not meaningfully distort visual working memory capacity estimates in the canonical change detection task. Front. Psychology 3:42. doi: 10.3389/fpsyg.2012.00042 This article was submitted to Frontiers in Cognition, a specialty of Frontiers in Psychology.

Copyright (C) 2012 Lin and Luck. This is an open-access article distributed under the terms of the Creative Commons Attribution Non Commercial License, which permits non-commercial use, distribution, and reproduction in other forums, provided the original authors and source are credited. 